\title{
RESULTS OBTAINED FROM OBSERVATIONS OF \\ LABORATORY PLASMAS
}

\section{W. LOCHTE-HOLTGREVEN}

Kiel University, Kiel, D.B.R.

In the visible part of the spectrum, the methods to solve astrophysical problems by laboratory experiments are well known. Electric arcs or shockwaves allow the production of plasmas of known parameters, from which measurements of oscillator strengths, of line broadening effects, and of line shifts are possible. However, there was a difficulty to introduce elements into laboratory light sources which have no volatile compounds. This was particularly difficult for the metals. This difficulty was overcome only recently by burning the electric arc in argon gas containing a few percent of chlorine. When this gas mixture is led over heated metal, some metalchloride is formed and fed into the arc. In this way, even a very small amount of metal can be investigated, so that the resonance lines of the metals are optically thin. As an example I would like to recall that during the past year Professor Richter at Kiel has succeeded in a redetermination of the absolute oscillator strengths of iron lines, which has allowed to correct the abundance of iron in the Sun's atmosphere. The abundance turned out to be ten times larger than assumed hitherto, leading to a new conception of the conditions in the photosphere (Kock and Richter, 1968; Garz and Kock, 1969; Garz et al., 1969a, b; Baschek et al., 1970). With this abundance, there is no difference in composition between the solar photosphere, the corona and meteorites. The method used for iron was applied to a number of other elements. The abundance of nickel is of special interest. The ratio of iron to nickel is again the same in the photosphere, in the corona, and in meteorites, being $1: 1.25 \pm 0.15$ (see Figure 1).

Figure 2 illustrates the difficulties in the determination of the oscillator strengths.

Here, the ratio of the measured oscillator strengths of two Ni lines is plotted against the concentration of the $\mathrm{Ni}$ atoms. The lower curve refers to line pairs having practically no difference in the excitation energy of the upper terms. In the two upper curves the ratio of the oscillator strength of a line starting from a level with high excitation energy to that of a line starting from a level with low excitation energy is given. The decrease of the curves with small concentration of $\mathrm{Ni}$ atoms indicates relatively too few $\mathrm{Ni}$ atoms in the high lying levels. This means that for small concentrations the Ni populations are not those expected in LTE. The argon gas, in which the arc is mainly burning is in LTE. The resonance lines of this gas are optically thick i.e. the light emitted in the resonance lines is re-absorbed and helps in the establishment of LTE. On the contrary, for nickel the resonance lines are optically thin. The energy is radiated out of the plasma, and nickel in small concentration does not participate in the equilibrium. Here may be a source of possible error in the determination of transition probabilities from laboratory plasmas when equilibrium is presupposed to exist. 


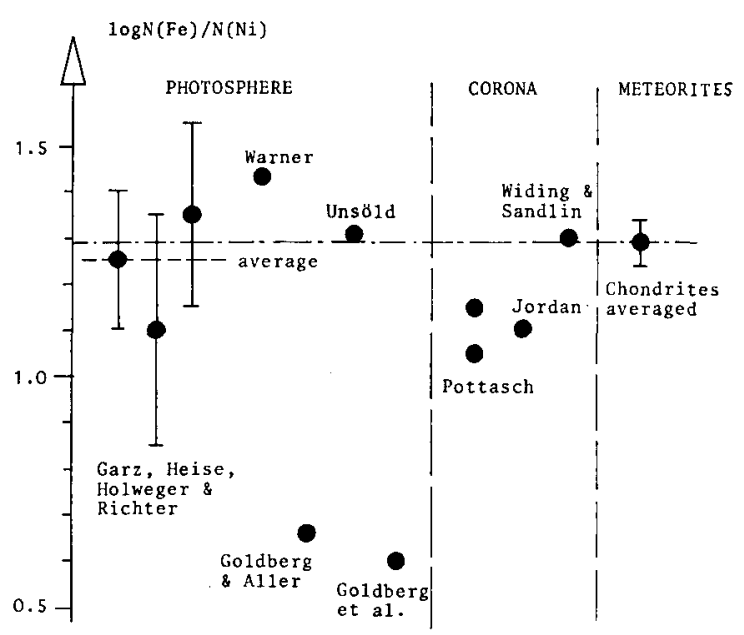

Fig. 1. Comparision of the iron to nickel abundance ratio in the photosphere, corona, and in meteorites.

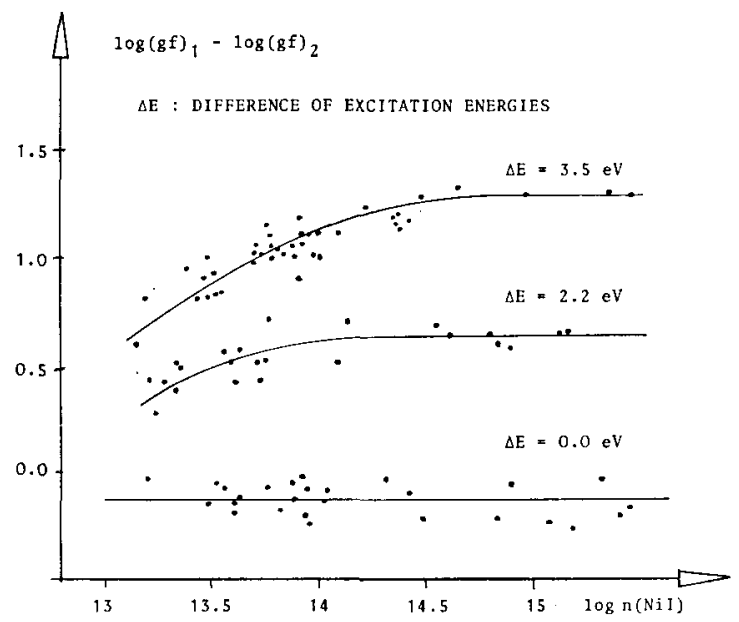

Fig. 2. The ratio of measured transition probabilities of five Ni lines plotted against the concentration of $\mathrm{Ni}$ in the laboratory light source. The parameter is the energy difference $\Delta E$ of the upper terms of the two lines.

Turning now to the UV part of the spectrum, the main problem has been the relative lack of data on the lines and levels belonging to the higher ionized atoms. Here much work has yet to be done. In the experimental identification the spectra obtained with beam foil spectroscopy and with laser produced plasmas have been of great help. For more quantitative work, the same difficulties arise as were anticipated in the visible part of the spectrum with regard to thermal equilibrium in the light source. A further difficulty was found in the relatively high temperatures necessary 
to excite the higher ions in the laboratory light sources. The high temperatures necessitate for calibration a standard light source of high temperature.

Dr Boldt, who died earlier in the year at Munich, used a wall stabilized carbon arc in argon gas as radiation standard at $14000 \mathrm{~K}$. Ogurzowa and Podmoshensky used a capillary spark as radiation standard at some $30000 \mathrm{~K}$. Hence, quantitative measurements in the far UV have become possible. The determination of the shape of the Lyman- $\alpha$ line and of the oscillator strengths of OI lines between $1100 \AA$ and $1800 \AA$ were among the first quantitative results of practical importance. Different from the work in the visible part of the spectrum, there are additional possibilities for quantitative investigation. In addition to photography or to counting of the light quanta emitted or absorbed in the spectral lines, there is the possibility of counting photo-ionized ions as soon as photoionization takes place. I would like to give examples of the measurement of absorption and also of the counting of ions, both made by Dr Lincke and his collaborators at Kiel. The absorption was studied using the mercury $\lambda=1126$ line. This line corresponds to the transition $5 d^{10} 6 s^{21} S_{0}-5 d^{9}$ $6 s^{2} 6{ }^{3} P_{1}$, which means that one of the $5 d$ electrons is excited to a level higher than the ionization limit. In the excited state, the atom may autoionize or else a line beyond the ionization limit may be emitted. The absorption line was previously assumed to be the strongest line of the neutral spectrum, $f$-values of 2 to 3 had been suggested. In the research of Lincke and Stredele (1970), a microwave lamp illuminated an absorption cell $30 \mathrm{~mm}$ long, closed with $\mathrm{LiF}$-windows. The temperature of the cell was varied between 50 and $98^{\circ} \mathrm{C}$ and the vapour density was taken from the vapour pressure curve. The absolute absorption is shown in Figure 3. The numerical integration yielded an oscillator strength of $f=0.530 \pm 0.032$. This result is remarkable, because the dispersion formula,

$$
n-1=\frac{e}{2 \pi m c^{2}} N_{0} \sum_{i} \frac{f_{i}}{v_{i}^{2}-v^{2}}
$$

with the resonance lines $\lambda=2537, f=0.0235$ and $\lambda=1850, f=1.18$, suggest the value $f=2$ to 3 for the line $1126.6 \AA$. With $f=0.53$ there is no possibility of representing the refraction of mercury vapour by a formula having three terms only. From this it becomes clear that calculations of $f$-values from the dispersion formula are quite unreliable.

It is certainly interesting to see the curve of growth for the mercury line at $1126 \AA$. The profile of this line (a line leading to autoionization) is so broad that the usual mechanisms of broadening are quite negligible. The curve shows proportionality with the number of absorbing atoms over almost 5 orders of magnitude (see Figure 4).

The other example concerns the transition probabilities of autoionizing lines from measurement of the photo-ions. I would like to point out that photoionization experiments are much more suited for quantitative measurements, because even very small absorption will always yield photo-ions. Therefore substances of very low vapour pressure can be investigated. A much larger range of absorption cross-sections 




Fig. 3. Quantitative measurement of the absorption cross-section and absorption profile of the $\mathrm{Hg}$ line at $1126 \AA$. The line profile is the difference between two photometer curves with and without mercury in the absorbing cell.

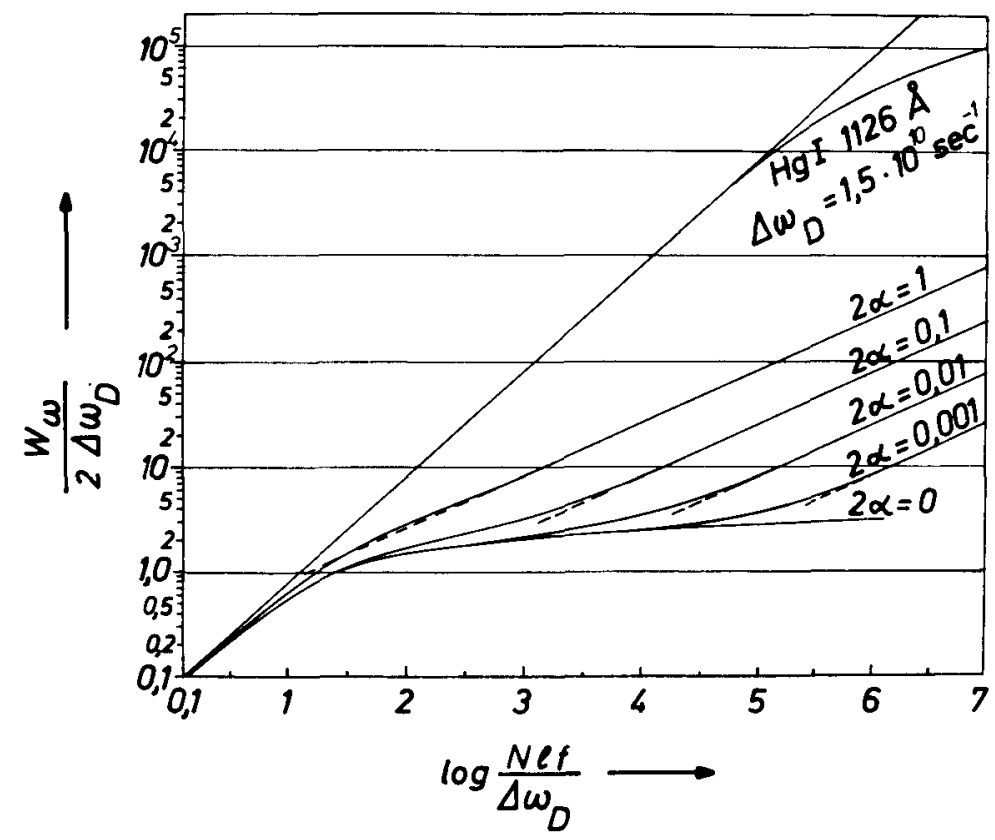

Fig. 4. The curve of growth for ordinary spectral lines and for the mercury $1126 \AA$ line leading to autoionization. 
is accessible. When the ions are investigated by mass spectroscopy the ionic species can be identified unambiguously and impurity lines can be eliminated. Autoionizing lines are well known from the research of Beutler, Garton $e t$ al. These lines are very numerous in the far UV region and can often be recognized easily by the peculiar Beutler-Fano shaped profiles.

The crossed beam technique has allowed the relative photoionization yields to be measured. The metal to be investigated was evaporated from a small graphite oven inside of an electrically heated tantalum tube (Lincke and Tegeder, 1970). Measurements of momentum and mass with a microbalancegave densities in the range of $10^{10}$ to $5 \times 10^{11}$ atoms $/ \mathrm{cm}^{3}$ in the beam. This beam was irradiated by a microwave discharge via a monochromator, which allowed the incident radiation to be scanned with a bandwidth of a few angstroms. The photo-ions produced were collected and accelerated through a quadrupole mass filter into an ionization chamber. In Figure 5 we see the relative ionization yield of AgI. The absolute values obtained are accurate to better than $20 \%$. These results may be compared with those of Paul and of Shenstone. The lines observed agree quite well in their wavelengths and intensities.

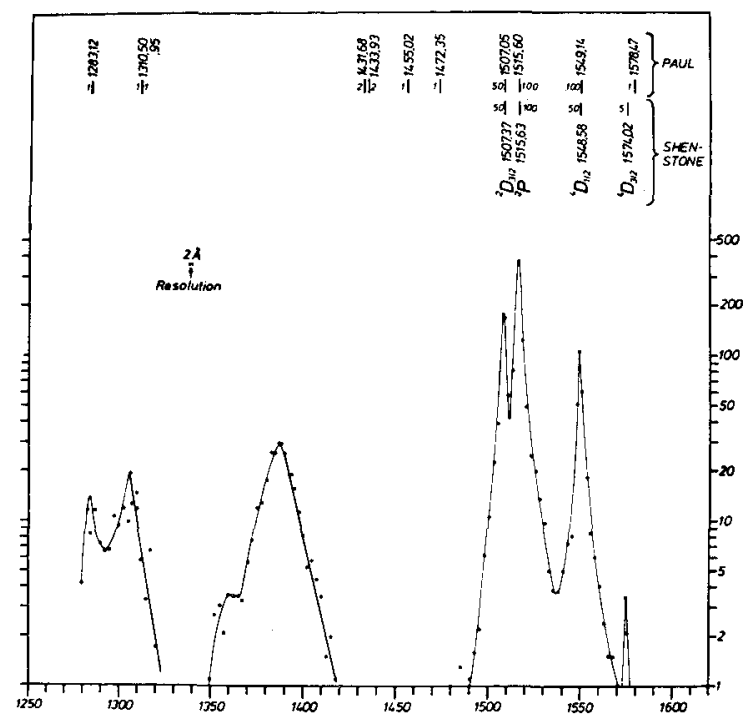

Fig. 5. Spectrum of the photo-ion yield from a beam of atomic silver. The previous results of Paul and of Shenstone are shown at the top.

In addition the lines which Shenstone indicated as dubious have been confirmed and a new emission feature at about $1387 \AA$ (which probably was too wide to be recognized before) has been observed. Hence, new atomic levels can be derived. Copper and gold have also been investigated in the same manner. Practically none of the levels involved have been known previously. 


\section{References}

Baschek, B., Garz, T., Holweger, H., and Richter, J.: 1970, Astron. Astrophys. 4, 229.

Garz, T. and Kock, M.: 1969, Astron. Astrophys. 2, 274.

Garz, T., Holweger, H., Kock, M., and Richter, J.: 1969a, Astron. Astrophys. $2,446$.

Garz, T., Kock, M., Richter, J., Baschek, B., Holweger, H., and Unsöld, A.: 1969b, Nature 223, 1254.

Kock, M. and Richter, J.: 1968, Z. Astrophys, 69, 180.

Lincke, R. and Stredele, B.: 1970, Z. Phys. 238, 164.

Lincke, R. and Tegeder, K.: 1970, in press.

\section{DISCUSSION}

G. W. Wares: I would like to comment on your remarks about methods for $f$-value measurement which are more recent than the use of unstabilized arcs and on the factor of ten increase in solar photospheric abundance of iron resulting from the new FeI $f$-value measurements made with the wall-stabilized arc in your laboratories. At the Air Force Cambridge Research Laboratories we have measured absolute $f$-values for FeI in our gas-driven shock tube, with temperatures measured ultrasonically. In his recent paper in Nature, J. E. Ross has used our $f$-values in advance of publication to calculate the photospheric abundance. Using a model atmosphere and the reproduction of observed line profiles, instead of the curve of growth method used at Kiel, he found approximately a factor of five increase in photospheric iron abundance. Using Meggers' NBS line intensities and our $f$-values or those of Kiel, for FeI, the calculated NBS free-burning arc temperature is $1000-2000 \mathrm{~K}$ higher than the value of $5100 \pm 110 \mathrm{~K}$ adopted by Corliss and Bozman on the basis of absorption $f$-values from King-type furnances, and used by them for the absolute $f$-values of 70 elements. Charles Corliss agrees that such a large temperature error existed. I wish to call attention to this situation in the principal tabulation of absolute $f$-values used by astrophysicists.

A. H. Gabriel: Professor Lochte-Holtgreven has drawn attention to the problems of auto-ionizing lines. As the charge on the ion increases, it becomes realistic to consider the possibilities of observing auto-ionizing lines in emission. In fact, the di-electronic satellites that we observe on the long wavelength side of the helium-like ion resonance lines are an example of such lines.

R.W.P.McWhirter: I should like to draw attention to some laboratory work of which the first comprehensive report has only just appeared and for this reason could hardly have been included in Professor Lochte-Holtgreven's review.

In this case and unlike the majority of the work to which Professor Lochte-Holtgreven referred, the observations are made on a plasma of very low density that is very far from being in local thermodynamic equilibrium. For such low density plasmas the atomic coefficient that determines the spectral intensity of a line is its excitation rate coefficient by electron collision from the ground or metastable level. The same coefficients are required for the interpretation of the majority of the emission lines from astronomical sources so that their accurate and reliable determination is a matter of primary importance to astrophysicists. Values of excitation rate coefficients are as essential to the analysis of non-LTE plasmas as $f$-values are to the analysis of LTE plasmas.

The experimental method is to measure the intensities of lines emitted by a plasma where electron temperature and density is well determined by the method of Thomson scattering of laser radiation. Complete details are given in the paper of Boland et al. (1970).

The results of these experimental measurements have been compared with values calculated theoretically and in general agreement is found within the claimed accuracy which is typically a factor 2 . The following list of papers gives access to those results of which I am aware.

Hinnov (1966) gives results for 15 lines in NeII-NevIII in the wavelength range 358-774 $\AA$. He used a stellarator plasma in which $\mathrm{N}_{e} \sim 10^{13} \mathrm{~cm}^{-3}, T_{e} \sim 10-50 \mathrm{eV}$, and the branching ratios method of calibration. His experimental accuracy is about a factor of 3 , and agreement within this factor is obtained when comparisons are made with the Bethe approximation.

\section{For H-like ions}

Jahoda et al. (1960) give results for the first six members of the Lyman series of OVII in the wavelength region $14 . \dot{4} 6 \AA-18.97 \AA$ observed with a Bragg crystal spectrometer. They used a Scylla I theta pinch with $N_{e}=7 \times 10^{16} \mathrm{~cm}^{-3}, T_{e}=300 \mathrm{eV}$. Their experimental accuracy was $15 \%$ for relative values and 
$65 \%$ for absolute values. There is agreement within the experimental errors, between their absolute results and the theoretical values obtained by McWhirter and Hearn, which were based on the Coulomb Born cross-sections due to Burgess. But for the relative values, particularly $\mathrm{Ly}-\alpha$ to $\mathrm{Ly}-\beta$ there is a discrepancy of a factor three. The explanation may be plasma opacity effects.

Hutcheon and McWhirter (1970, to be published). They give results for CVI and N vII, for Ly- $\alpha$ and Ly- $\beta$ only, in the wavelength range $20.9 \AA-33.7 \AA$. They used a grazing incidence spectrometer with X-ray tube calibration, and a theta-pinch with $N_{e}=2 \times 10^{16} \mathrm{~cm}^{-3}, T_{e}=150 \mathrm{eV}$. Their experimental accuracy is $10 \%$ for intensities and $10 \%$ for $T_{e}$, the total being about $20 \%$. The observed ratio of Ly- $\alpha$ to Ly- $\beta$ agrees with the theory within $25 \%$. (Preliminary result) The remaining discrepancy may be due to opacity.

\section{For He-like ions}

Elton and Koppendorfer, (1967) give the relative intensities of the OvII lines at 21.6 and $21.8 \AA$ and the absolute intensities of the $2 s-2 p$ triplet at $1623-1640 \AA$. They use Seya Namioka and grazing-incidence instruments, with a theta-pinch with $N_{e}=6 \times 10^{16} \mathrm{~cm}^{-3}, T_{e}=250 \mathrm{eV}$. Their experimental accuracy is about a factor of 2 .

Kunze et al. (1968) give results for the $\mathrm{Cv}$ lines at 40.3 and $40.7 \AA$, and the $O$ vir lines at 21.6 and $21.8 \AA$. They give relative intensities only, and also the absolute intensity of the $\mathrm{Cv}$ line at $2270 \AA$. A Bragg crystal spectrometer was used with a theta-pinch with $N_{e}=4 \times 10^{15} \mathrm{~cm}^{-3}, T_{e}=220 \mathrm{eV}$. Their experimental accuracy was about $25 \%$ and $40 \%$ respectively. The singlet excitation rate coefficients agree with the $\bar{g}$ formula to within the experimental accuracy of $40 \%$. There are no theoretical results with which to compare their other results for $\mathrm{Cv}$.

\section{For Li-like ions}

Boland et al. (1970) give results for lines of $\mathrm{NV}$ in the region 162-1234 $\AA$. They used a grazing incidence spectrometer and the branching ratios method for the calibration. The Zeta plasma device, with $N_{e}=10^{14} \mathrm{~cm}^{-1}, T_{e}=18 \mathrm{eV}$ was used. Their experimental accuracy is $\pm 20 \%$ for the relative values, and $\pm 50 \%$ for the absolute values. They find agreement to within the experimental errors with the theoretical values due to Burke.

Kunze and Johnston (1969, to be published) give results for Nevis lines in the region 67.4-770.4 $\AA$. They used grazing and normal incidence spectrometers and the branching ratios method of calibration. They used a theta-pinch, with $N_{e}=3.5 \times 10^{15} \mathrm{~cm}^{-3}, T_{e}=245 \mathrm{eV}$. Their experimental accuracy is about a factor of two, and they make no special claim for the relative accuracy. Their results agree with Bely's Coulomb-Born calculations and with an extrapolation of Burke's Nv results, except for $2 s-4 p$ where the measured value is more than a factor of two too small.

\section{For Be-like ions}

Tondello and McWhirter (to be published), have results for Nevis lines in the region 75-560 $\AA$. They use a grazing incidence spectrometer and the branching ratios method of calibration via an intermediate substandard. They use a theta-pinch with $N_{e}=5.5 \times 10^{15} \mathrm{~cm}^{-3}, T_{e}=2.1 \times 10^{6} \mathrm{~K}$. Their experimental accuracy is about $80 \%$ for absolute values and $50 \%$ for relative values. Their results are within a factor of two of the values predicted by Eissner's cross-sections, except for the $2 s 2 p^{3} P$ $2 s 3 d^{3} D$ transition where the experimental value is about a sixth of the theoretical value.

\section{References}

Boland, B. C., Jahoda, V. C., Jones, T. J. L., and McWhirter, R. W. P.: 1970, J. Phys. B. (2) 3, 1134. Elton, R. C. and Koppendorfer, W. W.: 1967, Phys. Rev. 160, 194.

Hinnov, E.: 1966, J. Opt. Soc. Am. 56, 1179.

Jahoda, F. C., Ribe, F. L., Sawyer, G. A., and McWhirter, R. W. P.: 1963, Proc. IV Conf. Ion Phon. Gases, 3, 347.

Kunze, H. J., Gabriel, A. H., and Griem, H. R.: 1968, Phys. Rev. 165, 267. 